\title{
PRIMARY PAPILLARY SEROUS ADENOCARCINOMA OF FALLOPIAN TUBE - A RARE CASE PRESENTATION.
}

\author{
M. Ramani, Puja Deshmukh, O.H.Radhika Krishna, V. Ratna Kumari
}

1. Professor. Department of Pathology, Niloufer hospital. Hyderbad, Andhra Pradesh.
2. Post Graduate. Department of Pathology, Niloufer hospital. Hyderbad, Andhra Pradesh.
3. Assistant Professor. Department of Pathology, Niloufer hospital. Hyderbad, Andhra Pradesh.
4. Professor. Department of Obstetrics \& Gynaecology, Niloufer hospital. Hyderbad, Andhra Pradesh.

\section{CORRESPONDING AUTHOR:}

Dr. M. Ramani,

Professor, Department of Pathology,

Niloufer hospital for women and child health,

Red hills, Hyderabad,

Andhra Pradesh.

E-mail: drmramani@sify.com

ABSTRACT: Primary fallopian tube carcinoma (PFTC) is rare and constitutes about $0.14 \%$ to $1.8 \%$ of all female genital (gynaecological) malignancies. It is so rare that it is often mistaken histologically and clinically for ovarian cancer. It arises in post menopausal women and typically presents with vaginal bleeding, pelvic pain and watery discharge.

We present a case of primary fallopian tube carcinoma in a 55yr old nulliparous female who presented with post menopausal bleeding.

KEYWORDS: primary fallopian tube carcinoma; papillary serous adenocarcinoma ; 55yr nulliparous female.

INTRODUCTION: Malignancy of the fallopian tube is extremely rare, accounting for less than $0.2 \%$ of malignancies in women annually. Primary fallopian tube carcinoma (PFTC) most frequently occurs between the fourth and sixth decades of life, with a median age of occurrence of 55 years. Histologically and clinically, it can be difficult to differentiate from ovarian cancer though the management is similar. Metastasis of fallopian tube carcinoma occurs via haematogenous, lymphatic, and peritoneal routes as well as via direct extension. This is a case of a post menopausal woman with primary fallopian tube carcinoma- papillary serous type.

CASE HISTORY: A 55-year-old nulliparous post-menopausal female was admitted for complaints of spotting per vaginum since 3 months. There was also a history of watery discharge and abdominal pain. She attained menopause 8 years back.

Clinical and relevant investigations were done and revealed: Ca 125 levels of $\mathbf{9 0 . 5}$ $\mathbf{U} / \mathbf{m l}$ (normal up to $35 \mathrm{U} / \mathrm{ml}$ ). Ultrasound showed a right tubo-ovarian mass measuring $8 \times 4 \times 3$ cms with hydrosalpinx, foci of irregular wall thickening, mural masses and multiple discrete echogenic papillary projections. Right ovary could not be visualized. Left ovary and tube were normal and accumulation of peritoneal fluid seen. CT scan showed that the lesion had cystic and non-enhancing areas. Rest of the abdominal organs were normal. Endometrial curetting was done and it showed cystically dilated endometrial glands. The patient underwent total abdominal hysterectomy with bilateral salpingo-oophorectomy. 
On Gross examination a cystic mass in the ampullary, infundibular and fimbrial region of the right fallopian tube measuring $10 \times 5 \times 3 \mathrm{cms}$ was seen. Right ovary was identified separately measuring $4 \times 1 \mathrm{cms}$.

Cut section of the mass showed cystic areas with brownish serous fluid and multiple papillary excrescences. Similar papillary foci were seen in the isthmic region of the fallopian tube. Left ovary was measuring $3.5 \times 0.5 \mathrm{cms}$, left tube measured $3 \times 1 \mathrm{cms}$, both were normal on gross examination. Uterus with cervix measured $8 \times 4 \times 2 \mathrm{cms}$. Uterus and cervix were nil remarkable. (fig. 1)

Microscopically, multiple sections from the tubal mass showed histological features of well differentiated adenocarcinoma (papillary serous type).The tumour was confined to the mucosa (endosalpinx), showed papillary pattern with fibrovascular cores. The tumour cells were cuboidal to columnar with moderate to scant amount of cytoplasm. Nuclei were round oval to mildly pleomorphic, vesicular with prominent nucleoli. (Fig. 2) The right and left ovary showed histology consistent with the post-menopausal changes and were free from tumour. Left tube was also free from tumour deposits. Sections from cervix showed chronic cervicitis with nabothian cysts. Microscopic examination of the sections from the uterus showed postmenopausal senile cystic endometrial atrophy and myohyperplasia. Omental fat and lymph nodes were free from tumour. Ascitic fluid was sent separately and showed predominantly lymphocytes and reactive mesothelial cells. Immunohistochemistry done showed positivity for Ck 7 and EMA, while Ck 20 was negative. (Fig. 3)

The post-operative period of the patient was uneventful. Subsequently, the patient turned up for follow-up only for a year.

DISCUSSION: Fallopian tube cancer is the least common of gynaecological malignancies. [1] It was first described by Renaud in 1847.It accounts for approximately $0.14 \%-1.8 \%$ of female genital malignancies. [2]

The age of the patient in this case is 55 years, which is comparable to all other studies. [3], [4], [5]. The distinctive presentation of intermittent, profuse, watery, clear to yellow (cholesterolrich) vaginal discharge (hydrops tubae profluens) is accompanied by colicky abdominal pain. The most common symptoms, which is seen in two thirds of patients is postmenopausal bleeding. The diagnosis is usually unsuspected preoperatively. [6], [7]

Carcinoma of the fallopian tube has a variable gross appearance usually causing enlargement of the tube. The tube may be retort shaped resembling a hydrosalpinx. Microscopically, the typical adenocarcinoma of the fallopian tube bears a very close resemblance to the serous adenocarcinoma of the ovary. About half of the tubal carcinomas are serous papillary, roughly a fourth are endometrioid, a fifth are transitional or undifferentiated and the remainder are of other rare epithelial cell types.[8] The papillary pattern becomes alveolar to solid as the grade increases. When tumour is confined to the lumen of the tube, the pattern is almost entirely papillary with tall columnar cells covering the epithelium.

PFTC* should be included in the differential diagnosis of any tubo-ovarian mass especially if the patient has clinical symptoms such as vaginal discharge or abnormal genital bleeding with negative diagnostic curettage. On CT scan, some tumours are completely solid and others are predominantly cystic and the latter contain papillary projections or solid regions. Although rare, PFTC must be considered in the differential diagnosis adnexal masses, and particularly in the presence of incomplete septations and a highly vascular, solid component. *PFTC : primary fallopian tube carcinoma 
Difficulties in diagnosis exist due to the similarities shared between fallopian tube carcinoma and epithelial ovarian carcinoma. Hu et al,[9] established diagnostic criteria to distinguish fallopian tube carcinoma from other primary tumours in 1950. This classification was modified in 1978 by Sedlis [10] which stands as:

- The tumour arises from the endosalpinx

- The histological pattern reproduces the epithelium of tubal mucosa

- Transition from benign to malignant epithelium is found

- The ovaries, uterus and cervix are either normal or with smaller tumour than that in the tube

PFTC spreads by local invasion, transluminal migration and via the lymphatics and the bloodstream. Patients with PFTC have a higher rate of retroperitoneal and distant metastases than those patients with epithelial ovarian cancer. The procedure of choice for treatment is total abdominal total hysterectomy, bilateral salpingo-oophorectomy, omentectomy, selective pelvic and para-aortic lymphadenectomy for any stage for fallopian tube carcinoma.

Immunohistochemical profile shown by fallopian tube carcinoma is Ca-125, CK 7, EMA positivity in tumour cells and CK 20 negativity.

In our case, the above mentioned criteria were fulfilled. First, the patient presented with the typical complaints of abdominal pain with intermittent bleeding and watery discharge and elevated Ca-125 levels in serum. The ultrasound showed a tubo-ovarian mass and the CT scan suggested a fallopian tube malignancy. Grossly the adenocarcinoma mass was present in the tube, microscopically limited to endosalpinx while both ovaries, other tube, uterus and cervix showed no evidence of tumour, satisfying most of the Hu et al criteria. The microscopic picture and the immunohistochemical profile together with the above details helped us arrive at the final diagnosis of primary papillary serous adenocarcinoma fallopian tube.

CONCLUSION: Fallopian tube cancer is a very rare genital cancer which is difficult to diagnose early and carries a poor prognosis. PFTC should be taken into account for making the differential diagnosis of a suspicious adnexal mass or a presumptive tubo-ovarian abscess in all post-menopausal women and also in the pre-menopausal women who fail to respond to antibiotic therapy and drainage. This case report highlights the vital role of clinical, pathological, and radiological correlation in the diagnosis of such malignancies.

\section{REFERENCES:}

1. Lialis A, Bakalianou K, Mpolsa E, et al. Fallopian tube malignancies.A retrospective clinical pathological study of 17 cases. J Obstet Gynecol 2008; 28 (7) : 93-95

2. Pectasides D, Pectasides E, Economopoulos T. Fallopian tube carcinoma: a review. Oncologist 2006; 11(8):902-12.

3. Hanton EM, Malkasian GD Jr, Dahlin DC, Pratt JH. Primary carcinoma of the fallopian tube. Am J Obstet Gynecol 1996;94:832-9

4. Eddy GL, Copeland LJ, Gershenson DM, Atkinson EN, Wharton JT, Rutledge FN. Fallopian tube carcinoma. Obstet Gynecol 1984; 64: 546-52.

5. Podratz KC, Podczaski ES, Gaffey TA, O'Brien PC, Schray MF, Malkasian GD Jr. Primary carcinoma of fallopian tube. Am J Obstet Gynecol 1986;154:1319-26

6. Stewart SL, Wlke JM, Faster SL. The incidence of primary fallopian tube cancer in the United States. Gynecol Oncol 2007;107(2):392-397 
7. King A, Seraj IM, Thrasher T, Slater J, Wagner RJ. Fallopian tube carcinoma clinicopathological study of 17 cases. Gynecol Oncol. 1989;33:351-355

8. Sternberg SS. Diagnostic surgical pathology. 4th ed. Philadelphia: Lippincott Williams \& Wilkins; 2004.

9. Hu C Y, Taymour M L, Hertig A T. Primary carcinoma of the fallopian tube. Am J Obstet Gynaeco1 1950; 59:58-67.

10. Sedlis A. Carcinoma of the fallopian tube. Surg Clin North Am1978; 58:121-9. 


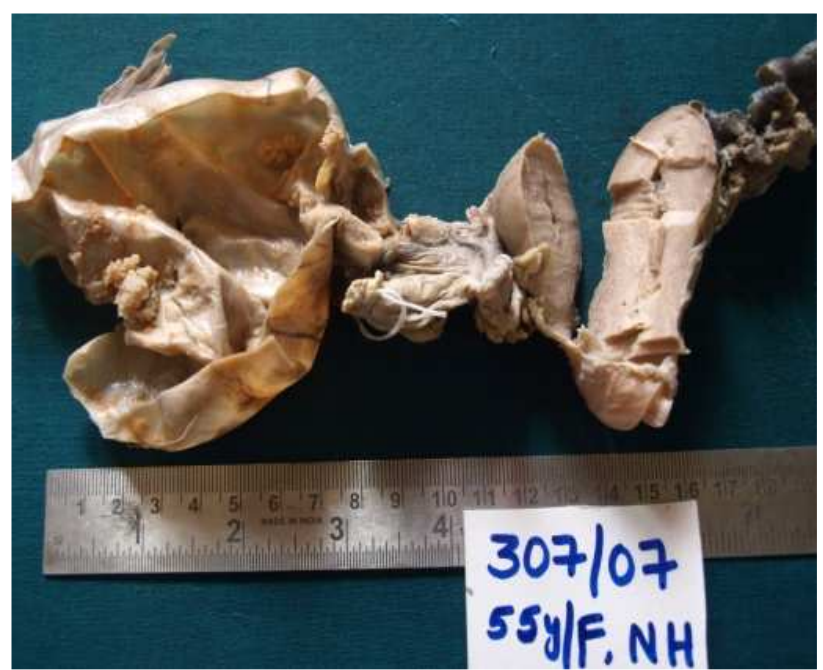

Fig1. Gross specimen showing cystic tubal mass with papillary excrescences

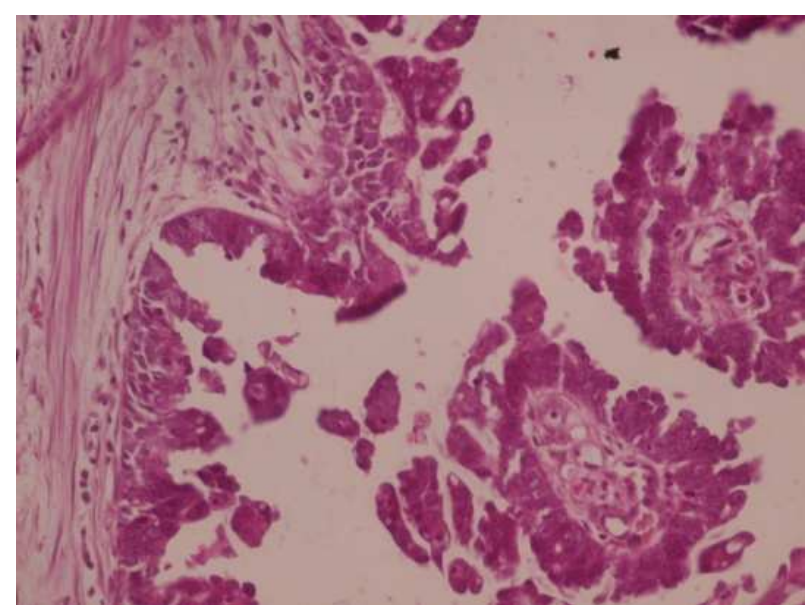

Fig2. (40x) Microscopically papillary projections lined by tumour cells



Fig 3. (40x)Immunohistochemistry Ck 7 positivity and CK 20 negativity seen in tumour cells 